\title{
Hydroxychloroquine in the treatment of COVID-19 disease: a systematic review and meta-analysis
}

Amahirany Manzo-Toledo, ${ }^{1}$ Rafael Torres-Rosas, ${ }^{2}$ Hugo Mendieta-Zerón, ${ }^{3}$ Lourdes Arriaga-Pizano, ${ }^{4}$ Liliana ArguetaFigueroa $^{5}$

pISSN: 0853-1773 - elSSN: 2252-8083 https://doi.org/10.13181/mji.oa.205012 Med J Indones. 2021;30:20-32

Received: September 09, 2020 Accepted: November 25, 2020 Published online: January 27, 2021

Authors' affiliations:

${ }^{1}$ Facultad de Odontología, Universidad Autónoma Benito Juárez de Oaxaca, Oaxaca, México, ${ }^{2}$ Laboratorio de Inmunología, Centro de Estudios en Ciencias de la Salud y la Enfermedad, Facultad de Odontología, Universidad Autónoma Benito Juárez de Oaxaca, Oaxaca, México, ${ }^{3}$ Facultad de Medicina, Universidad Autónoma del Estado de México, Toluca de Lerdo, México, ${ }^{4}$ Unidad de Investigación Médica en Inmunoquímica, Hospital de Especialidades Bernardo Sepúlveda, Centro Médico Nacional Siglo XXI, Instituto Mexicano del Seguro Social, Ciudad de México, México, ${ }^{5}$ CátedrasConacyt - Facultad de Odontología, Universidad Autónoma Benito Juárez de Oaxaca, México

\section{Corresponding author:}

Liliana Argueta-Figueroa

Cátedras-Conacyt - Facultad de Odontología, Universidad Autónoma Benito Juárez de Oaxaca. Av. Universidad $\mathrm{S} / \mathrm{N}$, Col. Cinco señores, Oaxaca de Juárez, Oaxaca, México, Zip Code: 68120 Tel/Fax: +52-1-951-44-8276

E-mail: liliana.argueta@conacyt.mx

\begin{abstract}
BACKGROUND Given the urgency of finding a specific treatment for coronavirus disease 2019 (COVID-19), several approaches have been carried out, including the use of chloroquine (CQ) and hydroxychloroquine (HCQ). This study was aimed to systematically evaluate the available evidence on the effectiveness of HCQ in the treatment of COVID-19 disease.
\end{abstract}

METHODS We searched 3 databases (PubMed, Google Scholar, and ClinicalTrials) until May 31, 2020 for clinical studies in patients diagnosed with COVID-19 comparing conventional treatment with and without $\mathrm{HCQ}$ combined with or without azithromycin. The risk of bias assessment and quality evaluation was carried out according to the Cochrane recommendations.

RESULTS 5 articles ( 1 randomized clinical trial [RCT], 1 non-RCT, and 3 cohort studies) were included. The main outcome measure in 2 articles was the virological conversion determined by reverse transcription-polymerase chain reaction; however, the findings of both studies were contrary. The main objective of the other studies was to determine the effects of HCQ on COVID-19 mortality, and the studies showed similar results. In general, the studies showed methodological limitations, risk of bias, and variable quality. A meta-analysis from 2,041 patients showed the odds ratio of mortality for patients having HCQ and standard care was 1.38 (95\% Cl 0.93-2.04).

CONCLUSIONS Considering the limited data available and the very low-to-moderate quality of the studies included in this systematic review, the evidence suggests that the HCQ administration does not decrease the risk of death from COVID-19.

KEYWORDS COVID-19, hydroxychloroquine, mortality, SARS-CoV-2
At present, there is no specific treatment for coronavirus disease 2019 (COVID-19), and given the urgency of finding specific forms of treatment, several approaches have been carried out, including the use of chloroquine (CQ) and hydroxychloroquine (HCQ). CQ is an aminoquinoline with an antimalarial effect, from which its hydroxyl analog, HCQ, is derived. The latter has shown a better tolerability and a higher clinical safety profile of HCQ than that of CQ during long-term use, allowing a higher daily dose, having fewer pharmacological interactions, ${ }^{1}$ and cost less. Likewise, in vitro and in vivo studies showed that HCQ had direct antiviral effects, which resulted from inhibition of the $\mathrm{pH}$-dependent step of the replication of various viruses; inhibition of lysosomal activity in antigen-presenting cells, as well as immunomodulatory capacity. Thus, this drug has been shown to have anti-severe acute respiratory syndrome coronavirus activity, in vitro and in vivo. ${ }^{2}$ 
However, although these experimental findings might support the possibility of use in humans, clinical data appeared to be conflicting and need to be interpreted with caution. Thus, it is crucial to conduct and analyze the literature about HCQ as a treatment for COVID-19. This study was aimed to systematically evaluate the available evidence on the effect of HCQ in the treatment of COVID-19 disease.

\section{METHODS}

This systematic review is based on the preferred reporting item for systematic review and meta-analysis (PRISMA) and Cochrane ${ }^{3}$ guidelines.

\section{Search strategy}

The eligibility criteria, keywords, and algorithms used for search strategy are shown in Table 1. The search included all studies published until May 31, 2020.

\section{Selection of studies and data extraction}

The process of selecting studies was carried out by two reviewers (AMT and LAF) independently, through the application of the eligibility criteria. A third reviewer (HMZ) resolved disagreements. Decisions about excluded studies were recorded with the reasons justifying their exclusion.

A standardized Microsoft Excel worksheet was prepared for the registration of the relevant data of all the studies included in the systematic review, such as participant demographics and baseline characteristics, the dosage, and frequency of administration of the drugs, statistical analysis, and primary outcome.

The study researchers were contacted for missing data or additional details via email. The data were recorded and processed in the Review Manager 5.4 program.

\section{Assessment of risk of bias}

Two independent reviewers (RTR and LAAP) were responsible for the risk of bias assessment. The tools

Table 1. Search strategy

\begin{tabular}{|c|c|}
\hline Strategy & Description \\
\hline Population & Patients diagnosed with COVID-19 \\
\hline Intervention & Hydroxychloroquine (HCQ) with or without azithromycin (AZI) \\
\hline Comparator & Placebo or symptomatic or conventional treatment \\
\hline Outcomes & Symptom relief, patient recovery, or seronegative or virological clearance or clinical cure \\
\hline Study design & Clinical trials, observational \\
\hline Eligibility criteria & $\begin{array}{l}\text { Studies involving adults and children with confirmation of the diagnosis for COVID-19 infection. In these } \\
\text { studies, the intervention was with HCQ alone or in combination with AZI and the control group was without } \\
\text { HCQ or with the administration of a placebo. Taking into account that the purpose of this review was to } \\
\text { analyze the reported evidence that has a minimum quality, only original articles from randomized, non- } \\
\text { randomized, and observational clinical studies, peer-reviewed, and accepted for publication }\end{array}$ \\
\hline Restrictions & $\begin{array}{l}\text { English and Spanish language. Peer-reviewed articles. Database of U.S. National Institutes of Health's } \\
\text { National Library of Medicine (PubMed) or Scientific Electronic Library Online (Scielo) }\end{array}$ \\
\hline Electronic database & Medline/PubMed, Google Scholar, and ClinicalTrials.gov \\
\hline Focused question & What is the effect of HCQ as a medication for the specific treatment against COVID-19? \\
\hline PubMed & $\begin{array}{l}\text { (“COVID-19"[Supplementary Concept] OR “COVID-19"[All Fields] OR "covid19"[All Fields]) AND } \\
\text { "treatment"[All Fields] AND ("hydroxychloroquine"[MeSH Terms] OR “hydroxychloroquine"[All Fields] OR } \\
\text { "HCQ" [All Fields]) AND ("outcome"[All Fields] OR "recovery"[All Fields]) AND ("clinical trial”[All Fields] OR } \\
\text { "clinical trials as topic"[MeSH Terms] OR "clinical trials"[All Fields]) }\end{array}$ \\
\hline Google Scholar & "COVID-19"+"treatment"+"hydroxychloroquine"+("outcome" or "recovery")+"clinical trial" \\
\hline ClinicalTrials.gov & $\begin{array}{l}\text { COVID -19 OR SARS-COV-2 | treatment | hydroxychloroquine | recovery OR outcome | Filters: Completed } \\
\text { Studies | Results }\end{array}$ \\
\hline
\end{tabular}

COVID-19=coronavirus disease 2019; SARS-CoV-2=severe acute respiratory syndrome coronavirus 2 
such as the Cochrane for assessing the risk of bias in randomized trials (RoB-2, Excel template with macros, online version $)^{4}$ and the risk of bias in non-randomized studies - of interventions (ROBINS-I) for observational studies, were used. ${ }^{5}$

\section{Quality assessment}

The quality of each study was performed considering the Grading of Recommendations Assessment, Development, and Evaluation (GRADE) criteria. ${ }^{6}$

\section{Strategy for data synthesis}

The qualitative and quantitative synthesis from the data of the included articles were performed. The heterogeneity between the measured effects from the studies was evaluated. The data were grouped according to a viral clearance and mortality. For the meta-analysis, the information collected from the selected studies were carefully analyzed to determine whether the studies can be grouped; however, studies that had a high risk of bias were not considered. Odds ratios were used from the individual studies and these were combined using a random-effects meta-analysis. Moreover, 95\% confidence intervals $(\mathrm{Cl})$ and two-sided values were calculated. The heterogeneity between the studies in terms of measures of effect was evaluated using the $I^{2}$ statistic and was considered an $\mathrm{l}^{2}$ value greater than $50 \%$ as being indicative of substantial heterogeneity.

\section{RESULTS}

Figure 1 shows the flow diagram of searching strategy. Of the five studies included in this systematic review, one randomized clinical trial (RCT), one non$\mathrm{RCT}$, and three cohort studies were found. The results of these studies added up to a total of 2,173 participants, of whom 1,207 patients were treated with HCQ and 966 patients were controls. The characteristics and results of the included studies can be seen in detail in Table 2. The risk of bias in the randomized clinical study was high (Figure 2a). In the observational studies, Gautret's study ${ }^{7}$ had a critical risk of bias, while the other three studies had a low risk of bias (Figure $2 b$ ). The quality assessment of the studies included in the systematic review is shown in Table 3.

Gautret et $\mathrm{al}^{7}$ carried out a study coordinated by the Institute of the University Hospital of

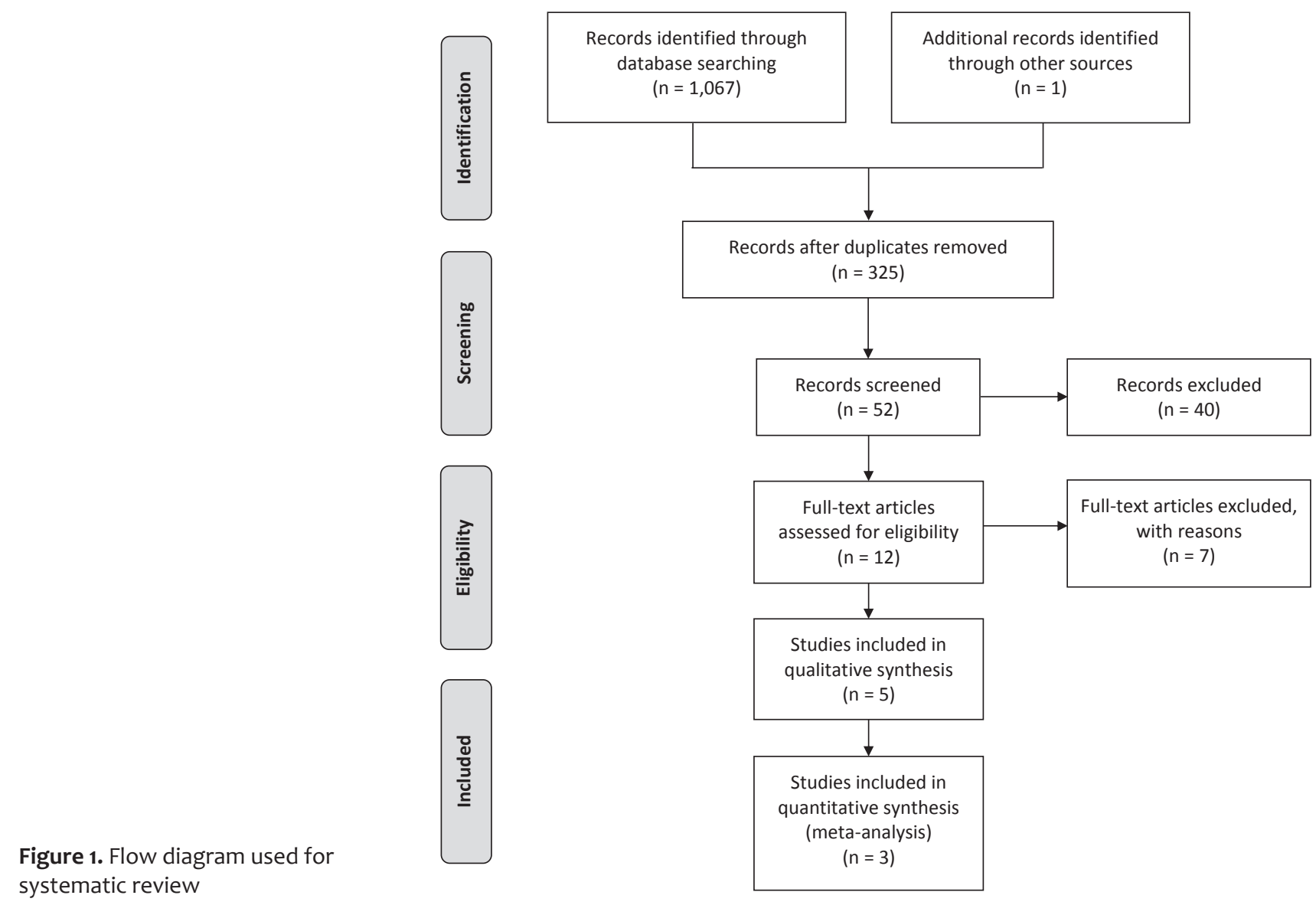

mji.ui.ac.id 


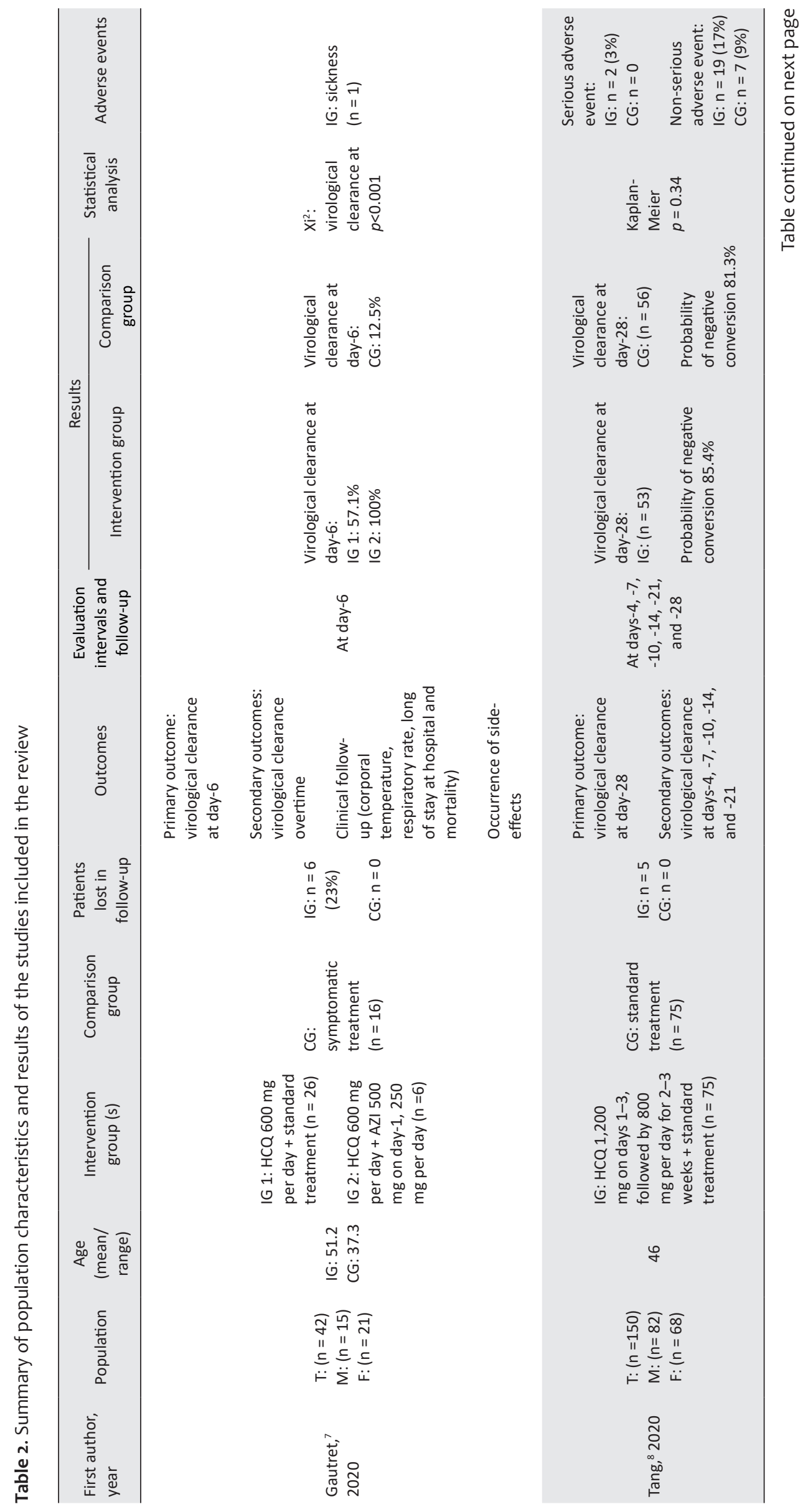




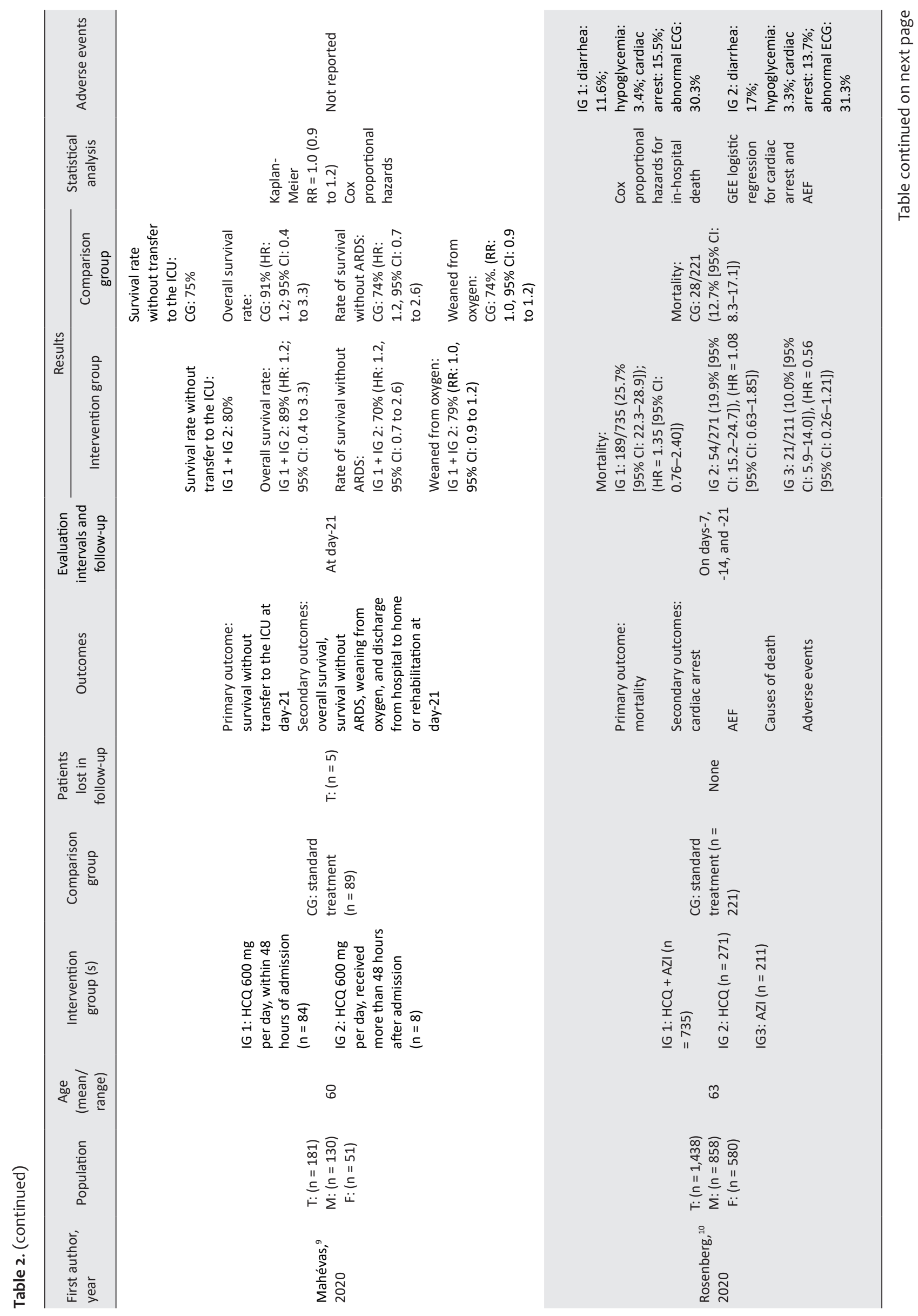




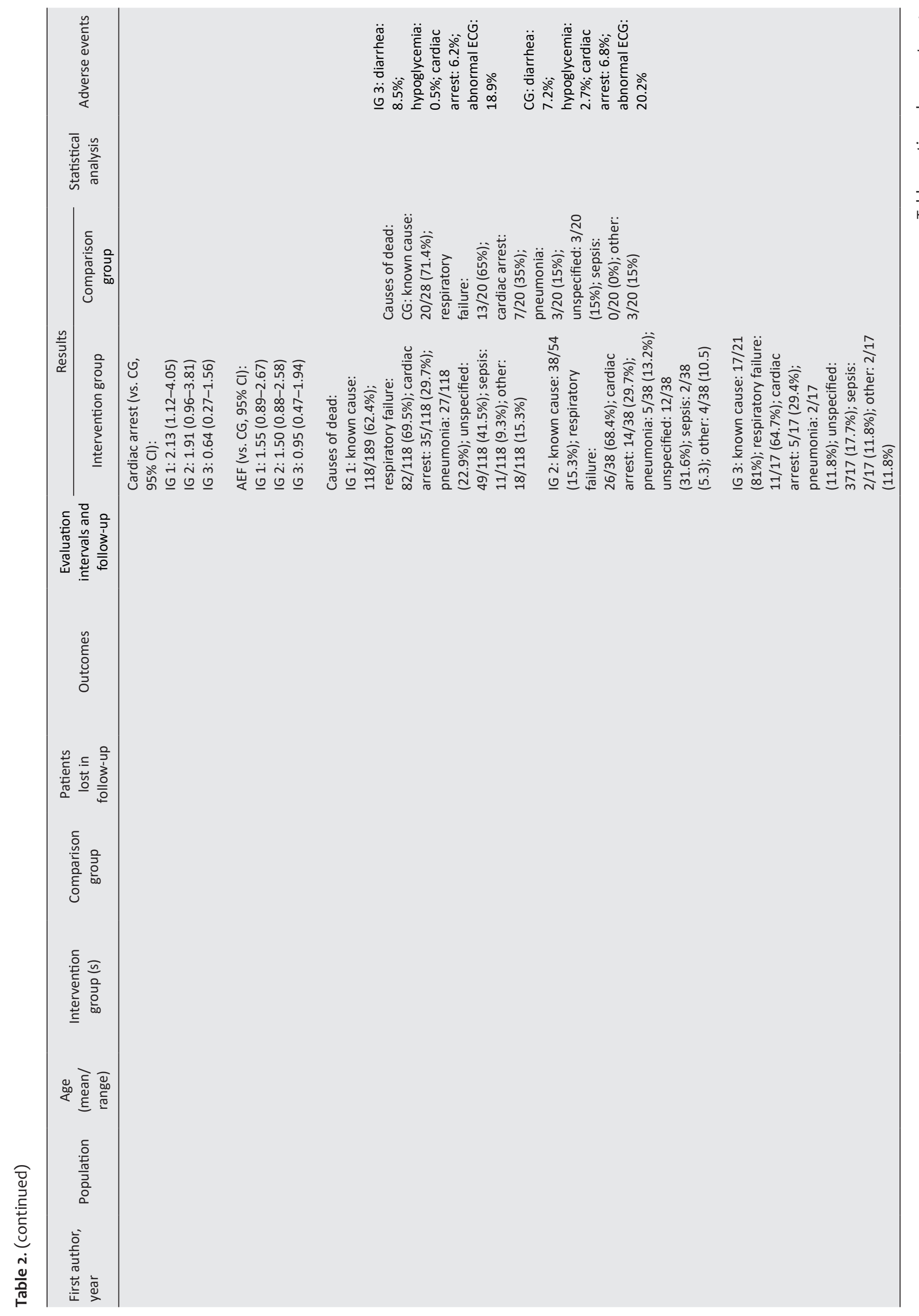




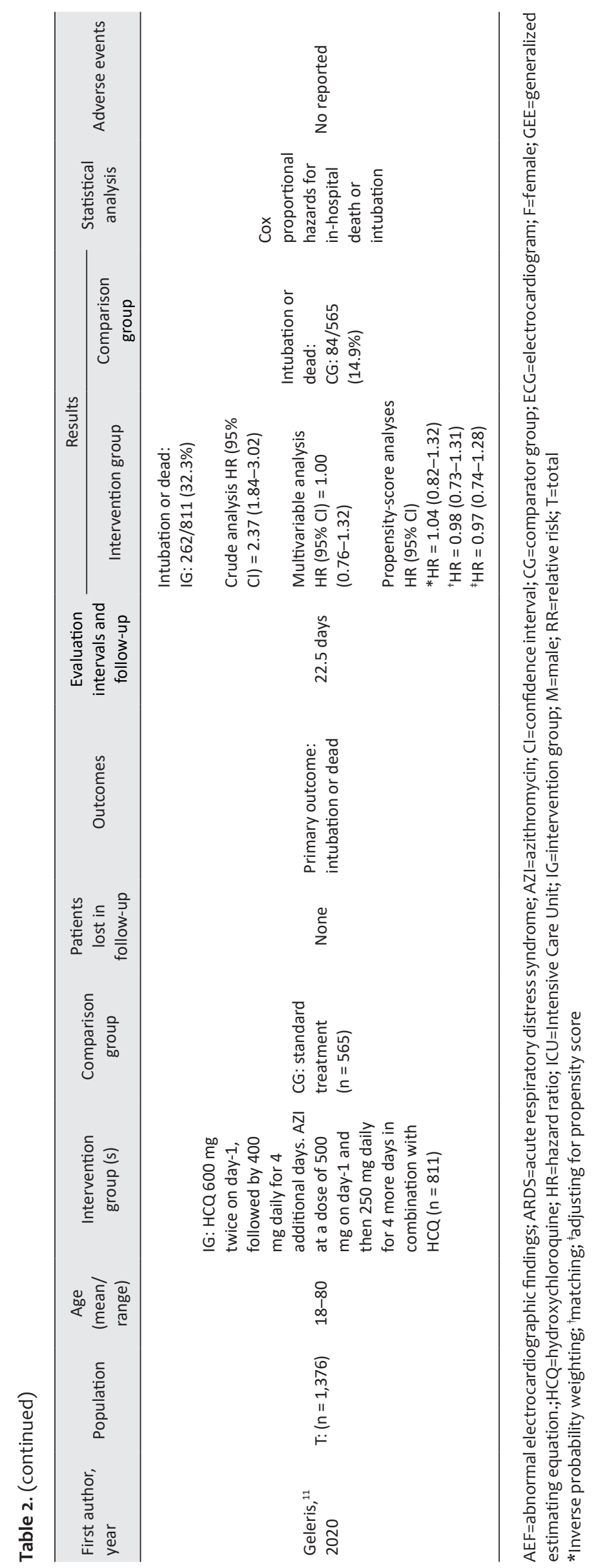


Studies with
intention-tointention-to-
treat
Unique ID Study ID Experimental

No hyr
Outcome

Negative virological convertion
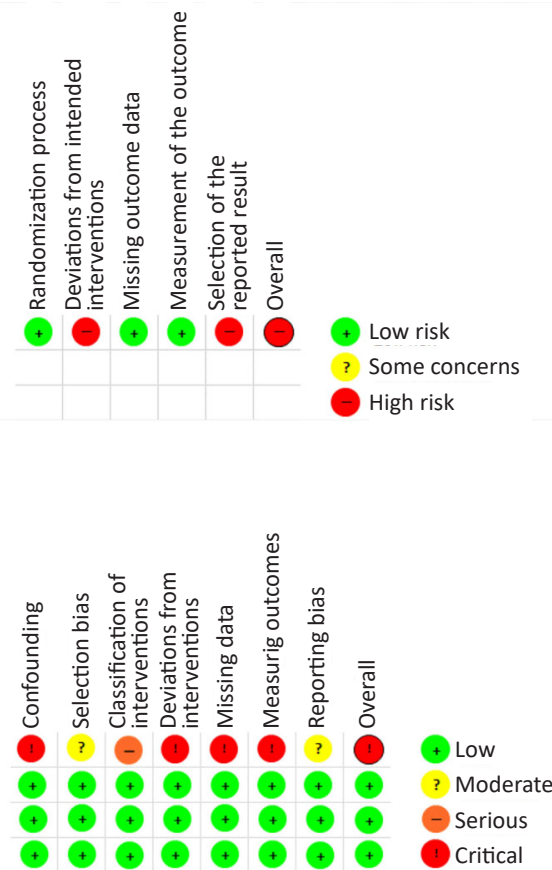

Figure 2. Risk of bias for individual studies from (a) risk of bias in randomized trials (RoB-2) assessment and (b) the risk of bias in non-randomized studies - of interventions (ROBINS-I) assessment

Mediterranée Infection in Marseille where they proposed a treatment with HCQ. Nevertheless, several points in the design of this study should be noted. The participants in the control group were not all taken from the same hospital in which the patients in the experimental group were treated; also, the controls in the center of Marseille were those patients who refused to receive the treatment or met any of the exclusion criteria. On the other hand, the population base from which the participants were taken had a significant difference in the severity between the patients treated and not treated with $\mathrm{HCQ}$. Moreover, the patients in the experimental group were older than those in the control group. The greatest risk of bias in this study was due to the lack of randomization in the intervention and the lack of blinding in all the people who participated in the study. The clinical condition of the participants in this study was categorized into: 1) asymptomatic; 2) upper respiratory tract infection; and 3) lower respiratory tract infection. The results did not show the raw data stratified by clinical condition; however, it was reported that the effect of the drug was greater in those with clinical signs of both upper and lower respiratory infection compared to asymptomatic patients $(p<0.05)$. In principle, it is unusual for asymptomatic patients to be hospitalized, suggesting doubts about whether there was another reason to be hospitalized. Other doubtful aspects of this study arose from the insufficient follow-up period and incomplete viral load determinations using reverse transcription-polymerase chain reaction, of which the results showed that only 2 of 16 patients had a negative seroconversion on day- 6 without mentioning that, in 5 of the 16 patients, the viral load determination was not performed. Finally, in this study, the secondary results were not reported, which raises more doubts about the reliability of this study. For the above reasons, the quality was very low due to the high risk of bias, as well as its inconsistency and imprecision.

Tang et $\mathrm{al}^{8}$ carried out a randomized study where they reported as a primary result that the overall probability of negative conversion at 28 days after the intervention, no statistical difference between groups was found. Also, no clinical improvement results were presented, since, within the study population, disease severity was heterogeneous. Besides, during the trial, they included the probability of symptom relief (resolution of fever, cough, sore throat, sputum production, and shortness of breath) as a secondary outcome, which was similar in patients assigned to the standard care with HCQ and without HCQ. It is noteworthy that not all secondary outcomes were recorded in the trial although they were included in the protocol. On the other hand, the dose of HCQ was adjusted in the patients when adverse events related to the medication occurred, indicating that 


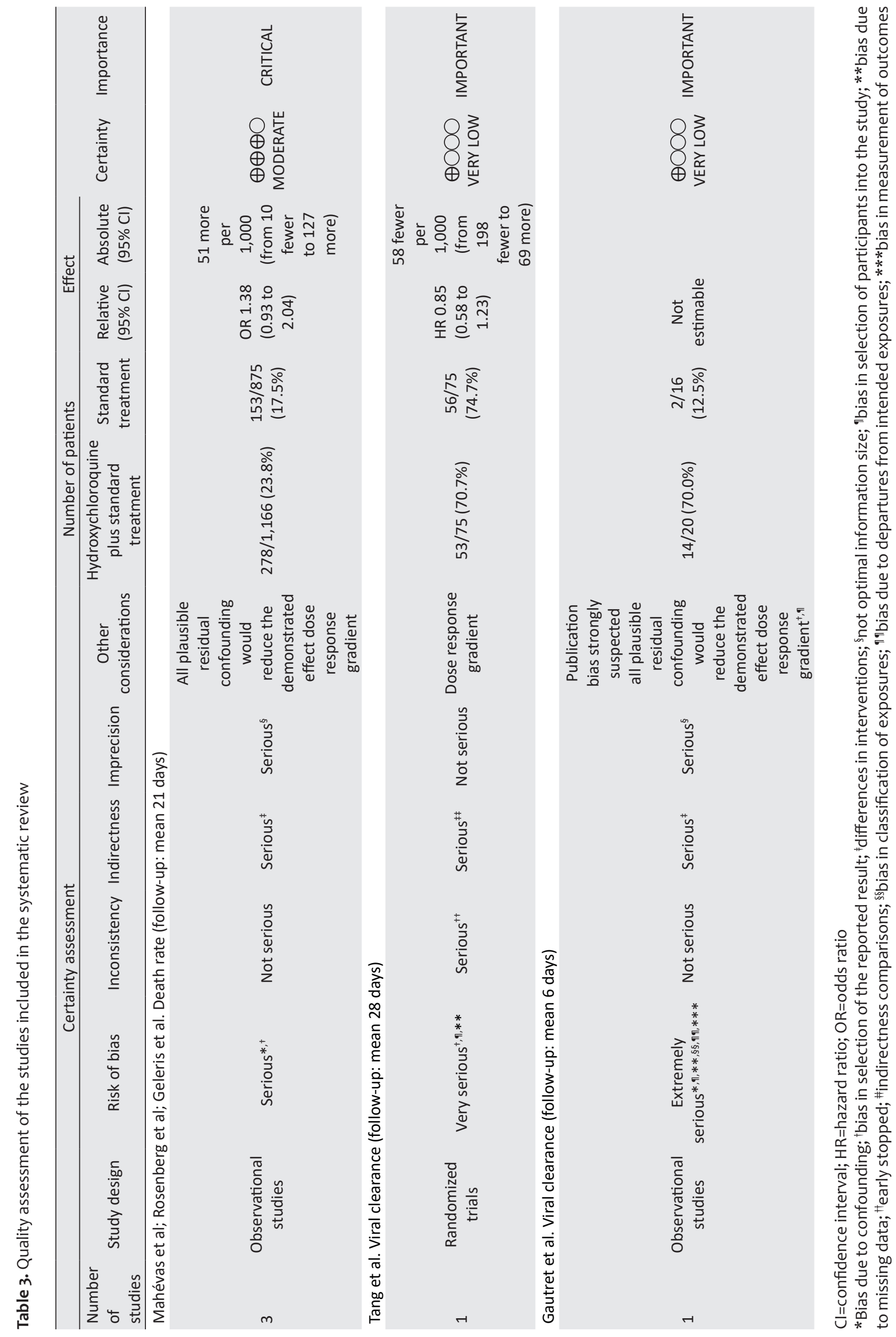




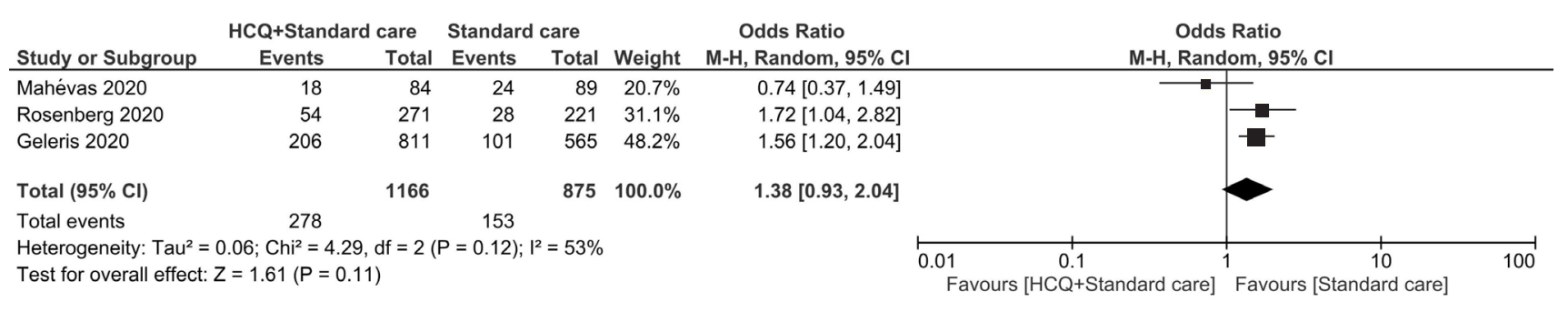

Figure 3. Meta-analysis of mortality for coronavirus disease 2019 (COVID-19) using hydroxychloroquine (HCQ) as treatment

the intervention was not the same for the members of the group with HCQ. The increased risk of study bias was due to the selection and randomization of the participants. In the study design, the intervention was planned to be assigned by intention to treat; however, this could not be achieved in all patients, as in the group receiving standard care plus $\mathrm{HCQ}$, six of the participants did not receive any doses of $\mathrm{HCQ}$ (three withdrew consent and three refused to be treated with HCQ) and were assigned to the standard care group. Also, one participant in the standard care group received HCQ because he presented with a severe clinical picture and was assigned to the standard care group plus HCQ. In addition to this, the study design was an open-label, so no type of blinding was applied. This study showed a high risk of bias, inconsistency, indirect results, and imprecision. As a consequence, the article had a very low quality.

Mahévas et al9 carried out an observational study comparing HCQ as a treatment for survival of patients with COVID-19 without a transfer to hospital intensive care units, in the intervention group and in the control group (not HCQ). After the intervention, Cox proportional hazards showed any risk differences between groups. An important point was that not all patients in the treatment group received the intervention at the same time since only some patients received HCQ 48 hours after admission and others within 48 hours of admission, so they considered these variables when adjusting the model. This study showed a low risk of bias.

Rosenberg et $\mathrm{al}^{10}$ conducted a retrospective cohort study, describing the association between HCQ with or without azithromycin (AZI) and clinical outcomes among hospitalized patients diagnosed with COVID-19. The result did not find that treatment with HCQ or AZI or in combination was different from not receiving any of the two drugs. The cohort consisted of a random sample of all the patients in 25 New York State hospitals, either one or both of the two experimental drugs were administered at the discretion of the treating physicians, but the assignation of the interventions occurred more frequently if the patients were sicker at the admission time, had comorbidities, or were elderly, which indicates that the baseline status was not homogeneous within groups. It should be noted that adverse effects, such as arrhythmias and cardiac arrest, could potentially appear before the initiation of the medication. Therefore, the onset of these events should be examined in relation to the time of administration of the medication. This study had a low risk of bias.

Geleris et al ${ }^{11}$ conducted an observational study, in which there was no significant association between HCQ use and intubation or death. This study reported limitations during the collection of clinical information from the population. There could be missing data for some variables and the possibility of inaccuracies in electronic records, such as the lack of documentation on smoking and pre-existing disease in some patients. It should be mentioned that patients in the cohort of this study were paired by the administration of AZI, tocilizumab, and remdesivir in some of the participants in the intervention group and the control group. This study had a low risk of bias, and the quality of the information for the mortality evaluation of these last articles was moderate due to the inconsistency, indirect results, and imprecision.

The results of the meta-analysis in the present review obtained of 2,041 events from the three studies suggested that mortality showed no differences between the patients who received HCQ and the controls, as seen in Figure 3. The funnel plot is not shown due to the small number of studies included in the meta-analysis.

\section{DISCUSSION}

Currently, despite the fact that there are no drugs approved by health organizations to treat COVID-19, health professionals have begun to recommend $\mathrm{HCQ}$, 
albeit without scientific support. ${ }^{12}$ In the present review, regarding the participants, the included studies were found with small sample sizes (Gautret et al, ${ }^{7}$ Tang et $\mathrm{al}^{8}{ }^{8}$ and Mahévas et $\mathrm{al}^{9}$ ), while only two studies had a sufficient number of participants to be considered an appropriate sample (Geleris et al ${ }^{11}$ and Rosenberg et $\left.\mathrm{al}^{10}\right)$. On the other hand, the illness, age, and comorbidities in the participants exhibited great heterogeneity (Mahévas et al, ${ }^{9}$ Tang et ${ }^{1},{ }^{8}$ and Rosenberg et $\mathrm{al}^{10}$ ).

Regarding the intervention, in two studies (Rosenberg et $\mathrm{al}^{10}$ and Geleris et $\mathrm{al}^{11}$ ), the administration of HCQ was in variable doses, routes, and time intervals. In contrast, in the other three studies, fixed doses of the drug were administered (Gautret et al, Tang et $\mathrm{al}^{8}{ }^{8}$ and Mahévas et $\mathrm{al}^{9}$ ). However, it seems that the differences in dose between studies showed no changes in the efficacy of the HCQ, except in Gautret et $\mathrm{al}^{7}$ study, which was at risk of critical bias and whose results were not reliable. On the other hand, in all studies, the comparator was those patients who received standard care without HCQ.

The use of $\mathrm{CQ} / \mathrm{HCQ}$ for the treatment of viral diseases is not a recent idea. The in vitro test results were promising; however, the experiments were performed under limited virus replication conditions. ${ }^{13}$ Later, in vivo experiments demonstrated that $\mathrm{CQ}$ did not show any positive effects against $\mathrm{H}_{3} \mathrm{~N}_{2}$ influenza virus. ${ }^{14}$ In the same way, in vitro studies claimed that CQ showed an effective anti-hepatitis $C$ virus effect due to the drug acts by targeting autophagic proteolysis. Likewise, $\mathrm{HCQ}$ also has been tested against the hepatitis $C$ virus, reporting a promising antiviral action. ${ }^{15}$ In addition, the antiviral effect of CQ has already been tested against HIV. In vitro studies seemed to suggest that the drug had broad-spectrum anti-HIV activity; nevertheless, in the animal experiments and few clinical trials, CQ exhibited no clinical benefit. ${ }^{16-19}$

Regarding the outcome, in the present systematic review, a meta-analysis using survival data with a random-effects model was performed because we considered the assumption that the studies were not all estimating the same intervention effect and had heterogeneity. The $\mathrm{X}^{2}$ statistic test shows homogeneity; however, the $I^{2}$ statistic indicates a moderate percentage of inconsistency; therefore, as $\mathrm{X}^{2}$ is not a test with high sensitivity, it is possible that the test may not have sufficient statistical power to detect heterogeneity. The choice between a fixed- effect and a random-effects meta-analysis should never be made on the basis of a specific cut point value from the statistical test for heterogeneity instead of the rationality from the causes of heterogeneity. ${ }^{20}$ Additionally, there is a clinical heterogeneity across the studies. Mahévas et $\mathrm{al}^{9}$ used data collected from routine care to assess the effectiveness of HCQ in patients with the same clinical severity of the disease (that required oxygen), whereas Rosenberg et al ${ }^{10}$ and Geleris et al, ${ }^{11}$ the illness severity was registered. Likewise, the dose of HCQ were different across the studies.

On the other hand, the validity of the cohort studies depends on the assumption that both groups are comparable with respect to other factors associated with the intervention or the outcome of interest. Therefore, in the survival analysis, the adjustment of the model is essential, considering the covariates, confounding variables, and the censored participants. ${ }^{21}$ Due to censoring, the Cox proportional hazards was suitable for data analysis to avoid bias due to missing data. ${ }^{22}$ In contrast, in the Gautret et $\mathrm{al}^{7}$ study, the follow-up time was insufficient and no such adjustment was carried out. Likewise, propensity score model matching is one of the strategies in the statistical analysis to reduce the possibility of section bias due to differences in the baseline characteristics of the participants. This pairing helps avoid attributing the differences in the results between the experimental and control groups to individual characteristics, which could have influenced the decision to administer HCQ to each participant, instead of showing real differences between the groups caused by the effect of the treatment itself. In all the observational studies included in the review, each multivariate multiple regression model was adjusted for the covariates, which was adequate. In these studies, similar results were found in the comparisons before adjusting the baseline characteristics, being a value close to 1 with a $\mathrm{Cl}$ that includes the unit (one). In the Geleris et al ${ }^{11}$ study, it was shown that without adjusting the participants' baseline characteristics, the effect on mortality from HCQ was overestimated. Raw data found a 2.37fold increased risk of dying with HCQ administration compared with those that no receive HCQ. In the Geleris et al $^{11}$ study,after adjusting the groups with the covariates and matching of the participants, the risk was 0.98 , with no significant difference in the risk between the two groups. 
Regarding adverse effects, a single-arm observational study found within the results that the administration of HCQ produced adverse effects such as gastrointestinal or cutaneous symptoms, headache, insomnia, and transient blurred vision presented mild adverse events in $2.35 \%$ of the patients, while in $97.6 \%$ of the patients the HCQ was well tolerated. In all, $0.04 \%$ of patients experienced more serious side effects, such as corrected QT interval (QTC) prolongation. ${ }^{23}$ One of the important findings within the study by Rosenberg et al $^{10}$ were adverse effects such as cardiac arrest and electrocardiographic findings (arrhythmias or prolonged QT fraction) in patients who received HCQ plus AZI or HCQ alone; these results were associated with pre-existing conditions such as hypertension, obesity, diabetes, elevation of liver enzymes, and abnormal kidney function. The metabolism of HCQ should also be considered, suggesting that toxicity is related to drug adherence in tissues. An interesting finding among the adverse effects was blurred vision, which, although it was considered a mild adverse effect, may be an indication of eye damage since HCQ is known to induce retinopathy. It binds to the melanin of the epithelial layer of the retina, resulting in loss of vision. Wolfe and Marmor ${ }^{24}$ conducted a study on retinal toxicity in patients with rheumatoid arthritis or systemic lupus erythematosus, who had been treated with HCQ; from a total of 3,995 patients, $6.5 \%$ discontinued treatment due to an eye pathology, of which $1.8 \%$ had retinal problems. The risk of toxicity was low in the first 7 years of exposure and was approximately five times higher after that period. Overall, the incidence of HCQ side effects appears to be relatively small when used for short intervals of time.

Several clinical trials have been suspended because preliminary results indicated that this drug provided no additional benefit or harm that the placebo for hospitalized COVID-19 patients, which is in agreement with the findings of this review. ${ }^{25} \mathrm{Also}$ the Solidarity Trial results in HCQ arm was suspended with 954 patients. The death rate ratio for HCQ was relative risk $=1.19$ (95\% Cl 0.89-1.59, $p=0.23$ ) and death/survival ratio for HCQ was 104/947 patients against its control (84/906) patients. In consequence, the evidence suggests that the HCQ is not a reliable treatment for COVID-19. ${ }^{26}$

The results of the present meta-analysis are in contrast with the review performed by Meo et $\mathrm{a}^{27}$ in which they reviewed in vitro studies, in vivo studies, original studies, clinical trials, and consensus reports, and concluded that CQ and HCQ could be useful against COVID-19. The potential deficiencies of this work are that there is no evidence that the PRISMA recommendations were followed, and the certainty of the evidence was considered the same for all types of study designs. Likewise, the risks of bias and quality assessment of the included studies were not carried out, and these are likely the reasons for the disparities in the results and conclusions reached. On the contrary, the present article performed a systematic review of the publications and included RCTs and observational studies. These methodological designs with patients showed more external validity and their results could be extrapolated to the clinical context. Additionally, most of these articles had a low risk of bias.

In contrast, a systematic review and meta-analysis made by Sarma et al, ${ }^{28}$ who found the virological cure outcome from two studies included, a high percentage of variation across studies attributable to heterogeneity $\left(1^{2}=73 \%\right)$. However, another issue in that analysis was identified. For all the previous reasons about the high risk of bias and low quality from the Gautret et al' study, their data were not appropriate to include in the meta-analysis. On the other hand, one of the problems of including small studies was the random error attributable to an insufficient sample, where the results could be scattered around the real effect and that can lead to overestimating or underestimating the effect. ${ }^{20}$ The two studies included in the meta-analysis had 57 and 40 participants, respectively. Besides, in such an analysis, a certain variation in the effect of the intervention is observed, and the inconsistency in the direction of the effect is particularly notable, so it can be misleading to quote an average value for the intervention effect. ${ }^{29}$ As a result, the analysis of the numerical data was misinterpreted.

Also, in the review carried out by Shah et $\mathrm{al}^{30}$ was reported the lack of robust evidence for $\mathrm{HCQ}$ and $\mathrm{CQ}$ as prophylactic drugs to prevent COVID-19. The main limitation was the design of the articles included in the review (three in vitro studies and two opinion articles), in consequence, they pointed out the need for data from RCTs to obtain reliable evidence. According to that, the use of $\mathrm{HCQ}$ is not recommendable as a prophylactic for COVID-19.

In conclusion, although it is essential to find a specific treatment for COVID-19 as soon as possible, shortcuts should not be taken in the methodological design to produce reliable data. Considering the 
limited data available and the low-to-moderate quality of the included studies in this systematic review, the evidence suggests that the HCQ administration does not decrease the risk of death from COVID-19.

\section{Conflict of Interest}

The authors affirm no conflict of interest in this study.

\section{Acknowledgment}

LAF thanks Cátedras-CONACYT program. AMR, RTR and LAF thanks the current administration of the Posgrade Division and the Dentistry School of Universidad Autónoma "Benito Juárez" de Oaxaca for their support. To Antonio Argueta-Solís (in memoriam) for their insights on the topic.

\section{Funding Sources}

None.

\section{REFERENCES}

1. Wu Z, McGoogan JM. Characteristics of and important lessons from the coronavirus disease 2019 (COVID-19) outbreak in China: summary of a report of 72,314 cases from the Chinese Center for Disease Control and Prevention. JAMA. 2020;323(13):1239-42.

2. Yao $X$, Ye F, Zhang $M$, Cui $C$, Huang $B$, Niu $P$, et al. In vitro antiviral activity and projection of optimized dosing design of hydroxychloroquine for the treatment of severe acute respiratory syndrome coronavirus 2 (SARS-CoV-2). Clin Infect Dis. 2020;71(15):732-9.

3. Cumpston M, Li T, Page MJ, Chandler J, Welch VA, Higgins JP, et al. Updated guidance for trusted systematic reviews: a new edition of the Cochrane Handbook for Systematic Reviews of Interventions. Cochrane Database Syst Rev. 2019;10:ED000142.

4. Sterne JA, Savović J, Page MJ, Elbers RG, Blencowe NS, Boutron I, et al. RoB 2: a revised tool for assessing risk of bias in randomised trials. BMJ. 2019;366:14898.

5. Sterne JA, Hernán MA, Reeves BC, Savović J, Berkman ND, Viswanathan $M$, et al. ROBINS-I: a tool for assessing risk of bias in non-randomised studies of interventions. BMJ. 2016;355:i4919.

6. Schünemann H, Brożek J, Guyatt G, Oxman A, editors. GRADE handbook [Internet]. 2013 [cited 2020 Apr 10]. Available from: https://gdt.gradepro.org/app/handbook/handbook.html.

7. Gautret P, Lagier JC, Parola P, Hoang VT, Meddeb L, Mailhe M, et al. Hydroxychloroquine and azithromycin as a treatment of COVID-19: results of an open-label non-randomized clinical trial. Int J Antimicrob Agents. 2020;56(1):105949.

8. Tang W, Cao Z, Han M, Wang Z, Chen J, Sun W, et al. Hydroxychloroquine in patients with mainly mild to moderate coronavirus disease 2019: open label, randomised controlled trial. BMJ. 2020;369:m1849.

9. Mahévas M, Tran VT, Roumier M, Chabrol A, Paule R, Guillaud $C$, et al. Clinical efficacy of hydroxychloroquine in patients with covid-19 pneumonia who require oxygen: observational comparative study using routine care data. BMJ. 2020;369:m1844.

10. Rosenberg ES, Dufort EM, Udo T, Wilberschied LA, Kumar J, Tesoriero J, et al. Association of treatment with hydroxychloroquine or azithromycin with in-hospital mortality in patients with COVID-19 in New York State. JAMA. 2020;323(24):2493-502.

11. Geleris J, Sun Y, Platt J, Zucker J, Baldwin M, Hripcsak G, et al. Observational study of hydroxychloroquine in hospitalized patients with Covid-19. N Engl J Med. 2020;382:2411-8.

12. Sharma A. Chloroquine paradox may cause more damage than help fight COVID-19. Microbes Infect. 2020;22(4):154-6.

13. Shibata M, Aoki H, Tsurumi T, Sugiura Y, Nishiyama Y, Suzuki S, et al. Mechanism of uncoating of influenza B virus in MDCK cells: action of chloroquine. J Gen Virol. 1983;64(Pt 5):1149-56.
14. Vigerust DJ, McCullers JA. Chloroquine is effective against influenza $A$ virus in vitro but not in vivo. Influenza Other Respir Viruses. 2007;1(5-6):189-92.

15. Helal GK, Gad MA, Abd-Ellah MF, Eid MS. Hydroxychloroquine augments early virological response to pegylated interferon plus ribavirin in genotype- 4 chronic hepatitis C patients. J Med Virol. 2016;88(12):2170-8.

16. Vaccari M, Fenizia C, Ma ZM, Hryniewicz A, Boasso A, Doster $M N$, et al. Transient increase of interferon-stimulated genes and no clinical benefit by chloroquine treatment during acute simian immunodeficiency virus infection of macaques. AIDS Res Hum Retroviruses. 2014;30(4):355-62.

17. Jacobson JM, Bosinger SE, Kang M, Belaunzaran-Zamudio P, Matining RM, Wilson CC, et al. The effect of chloroquine on immune activation and interferon signatures associated with HIV-1. AIDS Res Hum Retroviruses. 2016;32(7):636-47.

18. Routy JP, Angel JB, Patel M, Kanagaratham C, Radzioch D, Kema I, et al. Assessment of chloroquine as a modulator of immune activation to improve CD4 recovery in immune nonresponding HIV-infected patients receiving antiretroviral therapy. HIV Med. 2015;16(1):48-56.

19. Keyaerts E, Li S, Vijgen L, Rysman E, Verbeeck J, Van Ranst M, et al. Antiviral activity of chloroquine against human coronavirus $\mathrm{OC} 43$ infection in newborn mice. Antimicrob Agents Chemother. 2009;53(8):3416-21.

20. Deeks J, Fellow J, Altman D. Analysing data and undertaking meta-analyses. In: Higgins JP, Thomas J, Chandler J, Cumpston M, Li T, Page MJ, et al, editors. Cochrane handbook for systematic reviews of interventions version 6.0 (updated July 2019). Cochrane; 2019 [cited 2020 Feb 21]. p. 243-96.

21. White IR, Royston P. Imputing missing covariate values for the Cox model. Stat Med. 2009;28(15):1982-98.

22. Pankratz VS, de Andrade M, Therneau TM. Random-effects Cox proportional hazards model: general variance components methods for time-to-event data. Genet Epidemiol. 2005;28(2):97-109.

23. Million $\mathrm{M}$, Lagier JC, Gautret $\mathrm{P}$, Colson $\mathrm{P}$, Fournier PE, Amrane S, et al. Early treatment of COVID-19 patients with hydroxychloroquine and azithromycin: a retrospective analysis of 1061 cases in Marseille, France. Travel Med Infect Dis. 2020;35:101738.

24. WolfeF, Marmor MF. Rates and predictors of hydroxychloroquine retinal toxicity in patients with rheumatoid arthritis and systemic lupus erythematosus. Arthritis Care Res. 2010;62(6):775-84.

25. National Institues of Health, U.S. Department of Health and Human Services. NIH halts clinical trial of hydroxychloroquine: study shows treatment does no harm, but provides no benefit [Internet]. 2020 [cited 2020 Aug 14]. Available from: https:// www.nih.gov/news-events/news-releases/nih-halts-clinical-trialhydroxychloroquine.

26. Pan $H$, Peto $R$, Karim QA, Alejandria M, Henao-Restrepo $\mathrm{AM}$, García $\mathrm{CH}$, et al. Repurposed antiviral drugs for COVID-19 -interim WHO SOLIDARITY trial results. medRxiv. 2020:2020.10.15.20209817.

27. Meo SA, Klonoff DC, Akram J. Efficacy of chloroquine and hydroxychloroquine in the treatment of COVID-19. Eur Rev Med Pharmacol Sci. 2020;24(8):4539-47.

28. Sarma P, Kaur H, Kumar H, Mahendru D, Avti P, Bhattacharyya $A$, et al. Virological and clinical cure in COVID-19 patients treated with hydroxychloroquine: a systematic review and metaanalysis. J Med Virol. 2020;92(7):776-85.

29. Deeks JJ, Altman DG, Bradburn MJ. Statistical methods for examining heterogeneity and combining results from several studies in meta-analysis. In: Egger M, Smith GD, Altman DG, editors. Systematic reviews in health care: meta-analysis in context. 2nd ed. London: BMJ Publishing Groups; 2001. p. 285-312.

30. Shah S, Das S, Jain A, Misra DP, Negi VS. A systematic review of the prophylactic role of chloroquine and hydroxychloroquine in coronavirus disease-19 (COVID-19). Int J Rheum Dis. 2020;23(5):613-9. 